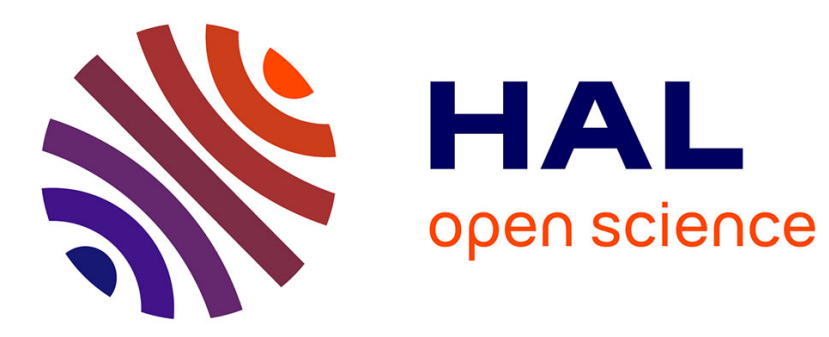

\title{
The Delaunay Hierarchy
}

Olivier Devillers

\section{- To cite this version:}

Olivier Devillers. The Delaunay Hierarchy. International Journal of Foundations of Computer Science, 2002, 13, pp.163-180. 10.1142/S0129054102001035 . inria-00166711

\section{HAL Id: inria-00166711 https://hal.inria.fr/inria-00166711}

Submitted on 8 Aug 2007

HAL is a multi-disciplinary open access archive for the deposit and dissemination of scientific research documents, whether they are published or not. The documents may come from teaching and research institutions in France or abroad, or from public or private research centers.
L'archive ouverte pluridisciplinaire HAL, est destinée au dépôt et à la diffusion de documents scientifiques de niveau recherche, publiés ou non, émanant des établissements d'enseignement et de recherche français ou étrangers, des laboratoires publics ou privés. 
published in Internat. J. Found. Comput. Sci., 13:163-180, 2002

\title{
The Delaunay Hierarchy
}

\author{
Olivier Devillers
}

\begin{abstract}
We propose a new data structure to compute the Delaunay triangulation of a set of points in the plane. It combines good worst case complexity, fast behavior on real data, small memory occupation and the possibility of fully dynamic insertions and deletions.

The location structure is organized into several levels. The lowest level just consists of the triangulation, then each level contains the triangulation of a small sample of the level below. Point location is done by walking in a triangulation to determine the nearest neighbor of the query at that level, then the walk restarts from that neighbor at the level below. Using a small subset $(3 \%)$ to sample a level allows a small memory occupation; the walk and the use of the nearest neighbor to change levels quickly locate the query.

keywords: computational geometry, geometric computing, randomized algorithms, Delaunay triangulation, dynamic algorithms.
\end{abstract}

\section{Introduction}

The computation of the Delaunay triangulation of a set of $n$ points in the plane is one of the classical problems in computational geometry and plenty of algorithms have been proposed to solve it.

These Delaunay algorithms can have different characteristics:

- Optimal on worst case data, i.e. $O(n \log n)$ time.

- Good complexity on random data only

- Randomized

- On-line vs off-line

In the current trade-off between algorithmic simplicity, practical efficiency and theoretical optimality, practitioners often opt for simplicity and practical

${ }^{0}$ INRIA, BP 93, 06902 Sophia Antipolis cedex, France. E-mail: Firstname.Lastname@sophia.inria.fr . This work was partially supported by ESPRIT LTR 21957 (CGAL). Preliminary version of that paper appeared in SoCG 98 [11] 
efficiency taking the risk of having bad performance on some special kind of data.

Our aim is to reconcile many of the above aspects, namely to obtain an incremental algorithm using simple data structures, having a good practical performance on realistic input and still having a provable $O(n \log n)$ expected randomized computation time in the worst case.

\section{Previous related work}

Our work is strongly related to some previous algorithms for Delaunay triangulation. All these algorithms are incremental and their complexity is randomized, they use some location structure to find where the new point is inserted, and then update the triangulation.

The first idea for a randomized incremental construction for the Delaunay triangulation [5] uses a location structure based on the history of the Delaunay triangulation: the Delaunay tree. Point $p_{i}$ is inserted at time $i$; to find the position of $p_{n}$ in the triangulation, $p_{n}$ is located in all the triangulations at times 1 to $n-1$; the location at time $i+1$ is deduced from the location at time $i$. This idea yields an expected $O(n \log n)$ complexity $[6,20]$ if the points are inserted in a random order. The drawbacks of this approach are the following: the location structure consists of the history of the construction and thus strongly depends on the insertion order, and the additional memory needed cannot be controlled. (The expected memory is proved to be $O(n)$ and is experimentally about twice the size of the final triangulation.)

Mulmuley [25] proposed a location structure independent of the insertion order. The structure has $O(\log n)$ levels, each level being a random sample of the level below. At each level, the Delaunay triangulation of the points is computed, and the overlapping triangles at different levels are linked to enable location of new points. This structure has the advantage of being independent of the order of insertion, of ensuring an $O\left(\log ^{2} n\right)$ location time for any point, and of allowing deletions in an easier way than the Delaunay tree [13]. However, the additional memory is still important and the location structure is not especially simple.

Mücke, Saias and Zhu [24] proposed a very simple structure and algorithm called jump and walk to handle triangulation of random points. The structure reduces to a random subset of $\sqrt[3]{n}$ points and pointers, from each of these points, to an incident triangle in the Delaunay triangulation. A new point is located by finding the nearest neighbor in the sample by brute force, and walking in the triangulation starting at that point. For evenly distributed points, the expected number of points in the zone of a point of the sample is $O\left(n^{\frac{2}{3}}\right)$ and since the complexity of the walk has the behavior of a square root [17], we get an $O(\sqrt[3]{n})$ time for locating a point and an $O\left(n^{\frac{4}{3}}\right)$ complexity for constructing the whole triangulation. The simplicity of that algorithm makes it competitive with many $O(n \log n)$ algorithms, but for some data (for example points on a curve) the complexity may increase to $O\left(n^{\frac{5}{3}}\right)$. 


\section{Overview}

Our approach uses a structure with levels similar to Mulmuley, but with simpler relations between levels. This allows a better control of the memory overhead. The transition between two levels is not direct as in Mulmuley but, similarly to Mücke, Saias and Zhu's algorithm, uses a walk to locate a point in a triangulation.

In Section 2 we present the algorithm, in Section 3 we prove that the expected complexity of constructing the Delaunay triangulation is $O(n \log n)$. The generalization to higher dimensions is explained in Section 4. In Section 5, the parameters of the data structure are then tuned to minimize the constant in the case of random points and are shown to yield an excellent behavior, we pay special attention to the comparison with the method of Mücke, Saias and Zhu. Finally we give some implementation remarks and practical results in Section 6.

\section{Algorithm}

Let $\mathcal{S}$ be a set of $n$ sites in the plane. The aim is to compute the Delaunay triangulation $\mathcal{D} \mathcal{T}_{\mathcal{S}}$ of $\mathcal{S}$ and to maintain it efficiently under insertions and deletions.

\subsection{The location structure}

The algorithm uses a data structure composed of different levels. Level $i$ contains the Delaunay triangulation $\mathcal{D} \mathcal{T}_{i}$ of a set of sites $\mathcal{S}_{i}$.

The sets $\mathcal{S}_{i}$ forms a decreasing sequence of random subsets of $\mathcal{S}$ based on a Bernoulli sampling technique [23, 26]:

$$
\begin{gathered}
\mathcal{S}=\mathcal{S}_{0} \supseteq \mathcal{S}_{1} \supseteq \mathcal{S}_{2} \supseteq \ldots \supseteq \mathcal{S}_{k-1} \supseteq \mathcal{S}_{k} \\
\left.\operatorname{Prob}\left(p \in \mathcal{S}_{i+1} \mid p \in \mathcal{S}_{i}\right)=\frac{1}{\alpha} \in\right] 0,1[.
\end{gathered}
$$

The data structure is fairly simple: it contains the points of $\mathcal{S}$ and the triangles of all the triangulations $\mathcal{D} \mathcal{T}_{i}$. A point $p \in \mathcal{S}$ such that $p \in \mathcal{S}_{i} \subseteq \ldots \subseteq$ $\mathcal{S}_{0}$ and $p \notin \mathcal{S}_{i+1}$ is said to be a vertex of level $i$ and has a link to a Delaunay triangle of $\mathcal{D} \mathcal{T}_{j}$ incident to $p$ for all $j$ between 0 and $i$. A triangle of $\mathcal{D} \mathcal{T}_{i}$ has links to its three neighbors in $\mathcal{D} \mathcal{T}_{i}$ and to its three vertices. The number $k$ of levels is not fixed; for each point, random trials decide its level, and the point with the highest level determines $k$.

\subsection{Location of a query}

To locate a query $q$, we start at a known vertex $v_{k+1}$ of the highest level $k$. Then we search for $v_{k}$, the vertex of $\mathcal{D} \mathcal{T}_{k}$ nearest to $q$. Since $v_{k}$ is also a vertex 
of $\mathcal{D} \mathcal{T}_{k-1}$, we search for $v_{k-1}$, the nearest neighbor of $q$ in $\mathcal{D} \mathcal{T}_{k-1}$, starting at $v_{k}$. The search is continued descending the different levels. At each level $i$, the nearest vertex $v_{i}$ of $q$ in $\mathcal{D} \mathcal{T}_{i}$ is determined.

At level $i$ the search for $v_{i}$ is carried out in three phases:

- First phase: from $v_{i+1}$, we have a link to a triangle of $\mathcal{D} \mathcal{T}_{i}$ having $v_{i+1}$ as a vertex. All triangles incident to $v_{i+1}$ are explored to find the triangle containing the segment $v_{i+1} q$.

- Second phase: all the triangles of $\mathcal{D} \mathcal{T}_{i}$ intersected by $v_{i+1} q$ are visited, walking along the segment $v_{i+1} q$ up to the triangle $t_{i}$ that contains $q$.

- Third phase: from $t_{i}$, we have to find the nearest neighbor $v_{i}$ of $q$. To this aim, we visit the triangles that may be intersected by line segment $q v_{i}$ : consider a visited triangle $w w^{\prime} w^{\prime \prime}$ of $\mathcal{D} \mathcal{T}_{i}$ such that $q$ lies within the circle $C^{\prime}$ through $w, w^{\prime}$ and $w^{\prime \prime}$. Assume without loss of generality than $|q w| \leq$ $\left|q w^{\prime}\right|,\left|q w^{\prime \prime}\right|$ and let $C$ be the circle of center $q$ through $w$, the relevant neighbors of $w w^{\prime} w^{\prime \prime}$ are visited. Irrelevant neighbors are determined by the following rules:

- Do not examine an already visited triangle.

- $C$ does not cut $C^{\prime}$ between $w^{\prime}$ and $w^{\prime \prime}$, thus the line segment $q v_{i}$ can not intersect $w^{\prime} w^{\prime \prime}$ and the neighbor of $w w^{\prime} w^{\prime \prime}$ through $w^{\prime} w^{\prime \prime}$ does not need to be examined (Figure 1a).

- if the absolute value of the angle $q w w^{\prime}$ (resp. $q w w^{\prime \prime}$ ) is greater than $\frac{\pi}{2}$ then $q v_{i}$ can not intersect $w w^{\prime}$ (resp. $w w^{\prime \prime}$ ) since $C$ (which contains $q v_{i}$ ) does not intersect $w w^{\prime}$ (resp. $w w^{\prime \prime}$ ). The neighbor of $w w^{\prime} w^{\prime \prime}$ through $w w^{\prime}$ (resp. $w w^{\prime \prime}$ ) does not need to be examined (Figure 1b).

This phase starts at triangle $t_{i}$ and selects among the vertices of the visited triangles the closest to $q$.

Figure 1c show the triangles visited by the different phases of the search.

\section{$2.3 \quad$ Updates}

Because of its simplicity, the data structure is fairly easy to update. Maintaining it dynamically provides a fully dynamic triangulation algorithm. The links between the different levels do not use any complicated data structure. The vertices just know a triangle at all levels in which they appear.

To delete a point from $\mathcal{S}$, just delete the corresponding vertex at all the levels where it appears, which can be done in time sensitive to $d$ the degree of that vertex. On average $d=6$ and thus some of the following algorithms can be used: a complicated algorithm [1] of deterministic complexity $O(d)$, a simple randomized $O(d)$ algorithm [8], solutions of complexity $O(d \log d)$ [12] or even 


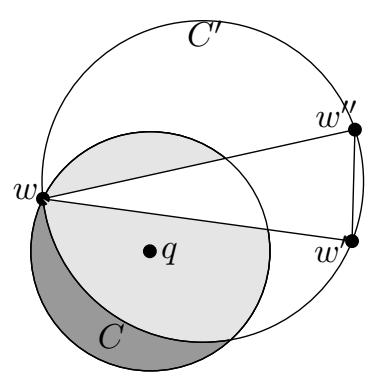

a)

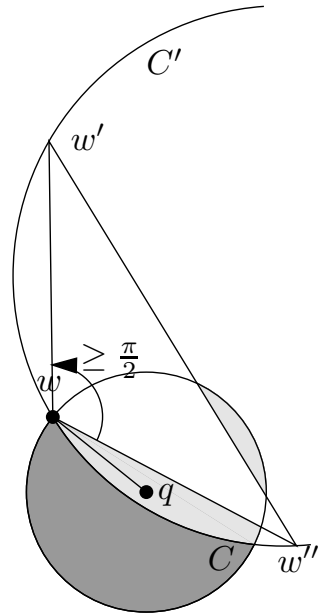

b)

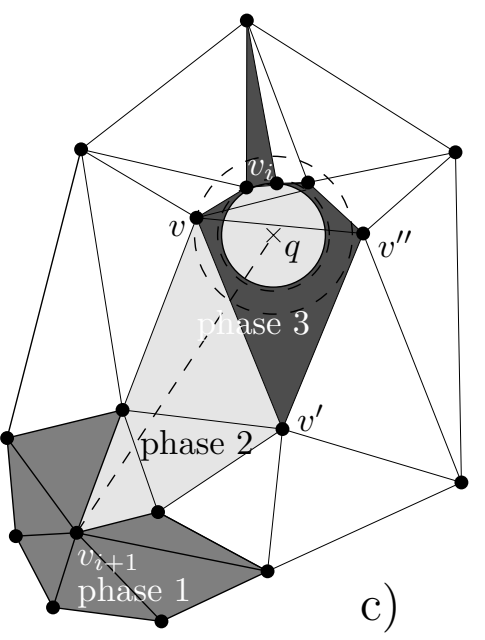

c)

Figure 1: Search for $v_{i}$.

simpler $O\left(d^{2}\right)$ algorithms. The simple sub-optimal solutions may be good in practice since $d$ is small on average.

Inserting a point in $\mathcal{S}$ reduces to locating it at all levels, computing its level $i$ and inserting the new vertex at all levels $j, 0 \leq j \leq i$ (which is sensitive to the degree of the new vertex since it is already located). The insertion at each level is done using a standard algorithm [21].

\section{Worst-case randomized analysis}

The analysis will rely on the randomization in the construction of the random subsets $\mathcal{S}_{i}$ and on the random order to insert the points of $\mathcal{S}$. In this section, no assumption applies to the data distribution, which can be the worst case distribution. As usual in theoretical computational geometry, we make only an asymptotic analysis and give rough upper bounds for the constants. In the next section, parameter $\alpha$ will be tuned to get a tight constant in the special case of evenly distributed points.

Let $\mathcal{S}$ be a set of $n$ points organized in the structure described in Section 2 and $q$ a point to be inserted in $\mathcal{S}$. Since we have assumed a random insertion order, $q$ is a random point of $\mathcal{S} \cup\{q\}$.

We denote $n_{i}=\left|\mathcal{S}_{i}\right|$ and $\mathcal{R}_{i}=\mathcal{S}_{i} \cup\{q\}$. 
Notice that, thanks to the random insertion order, $q$ is a random element of $\mathcal{R}_{i}$ : an element $v$ of $\mathcal{R}_{i}$ is equal to $q$ with probability $\frac{1}{n_{i}+1} . \mathcal{R}_{i}$ can be considered as a random subset of $\mathcal{R}_{i-1}$, indeed, given an element $v$ of $\mathcal{R}_{i-1}$, it belongs to $\mathcal{R}_{i}$ with probability $\frac{1}{n_{i-1}+1}+\frac{n_{i-1} \mid}{n_{i-1}+1} \frac{1}{\alpha}$ (the first term is the probability that $v=q$ and the second term is the probability that $v \in \mathcal{S}_{i}$ ). In the sequel, we denote by $E(X)$ the expected value of some quantity $X$, the expectation is on the whole randomization process, that is, $q$ is random in $\mathcal{R}_{i}$ and $\mathcal{S}_{i}$ is a random subset of $\mathcal{S}_{i-1}$.

The cost of exploring all the triangles incident to $v_{i+1}$ at the first phase of the walk of level $i$ is the degree of $v_{i+1}$ in $\mathcal{D} \mathcal{T}_{i}$. The cost of the second phase is the number of triangles intersected by segment $v_{i+1} q$. The cost of the third phase is the number of visited triangles during the search of $v_{i}$ from $t_{i}$.

Lemma 1 The expected degree of $v_{i}$ in $\mathcal{D} \mathcal{T}_{i-1}$ is $O(1)$.

Proof: The difficulty is that $v_{i}$ is not a random point in $\mathcal{R}_{i}$ but the nearest neighbor $N N(q)$ of a random point $q$ in $\mathcal{R}_{i}$. We will use the fact that $q v_{i}$ is an edge of $\mathcal{N} \mathcal{N}$, the nearest neighbor graph of $\mathcal{R}_{i}$, which has maximum degree 6 [27]. We denote by $d_{\mathcal{G}}^{\circ}(v)$ the degree of $v$ in some graph $\mathcal{G}$.

$$
\begin{aligned}
E\left(d_{\mathcal{D} \mathcal{T}_{i-1}}^{\circ}(N N(q))\right) & =E\left(\frac{1}{\left|\mathcal{R}_{i}\right|} \sum_{q \in \mathcal{R}_{i}} d_{\mathcal{D} \mathcal{T}_{i-1}}^{\circ}(N N(q))\right) \\
& =E\left(\frac{1}{\left|\mathcal{R}_{i}\right|} \sum_{q \in \mathcal{R}_{i}} d_{\mathcal{D} \mathcal{T}_{\mathcal{R}_{i-1}}^{\circ}}^{\circ}(q)+d_{\left.\mathcal{D} \mathcal{T}_{\mathcal{R}_{i-1}}^{\circ}(N N(q))\right)}\right. \\
& \leq 6+E\left(\frac{1}{\left|\mathcal{R}_{i}\right|} \sum_{v, q \in \mathcal{R}_{i}, v=N N(q)} d_{\mathcal{D} \mathcal{T}_{\mathcal{R}_{i-1}}^{\circ}}^{\circ}(v)\right) \\
& \leq 6+E\left(\frac{1}{\left|\mathcal{R}_{i}\right|} \sum_{v \in \mathcal{R}_{i}} d_{\mathcal{N \mathcal { N }}}^{\circ}(v) d_{\left.\mathcal{D} \mathcal{T}_{\mathcal{R}_{i-1}}^{\circ}(v)\right)}\right. \\
& \leq 6+E\left(\frac{1}{\left|\mathcal{R}_{i}\right|} \sum_{v \in \mathcal{R}_{i}} 6 d_{\mathcal{D} \mathcal{T}_{\mathcal{R}_{i-1}}}^{\circ}(v)\right) \\
& \leq 6+36 \leq 42
\end{aligned}
$$

Lemma 2 Given $w \in \mathcal{R}_{i}$, the expected number of vertices $q$ of $\mathcal{R}_{i}$ such that $w$ belongs to the disk of center $q$ and passing through the nearest neighbor of $q$ in $\mathcal{R}_{i+1}$ is less than $6 \alpha$. 


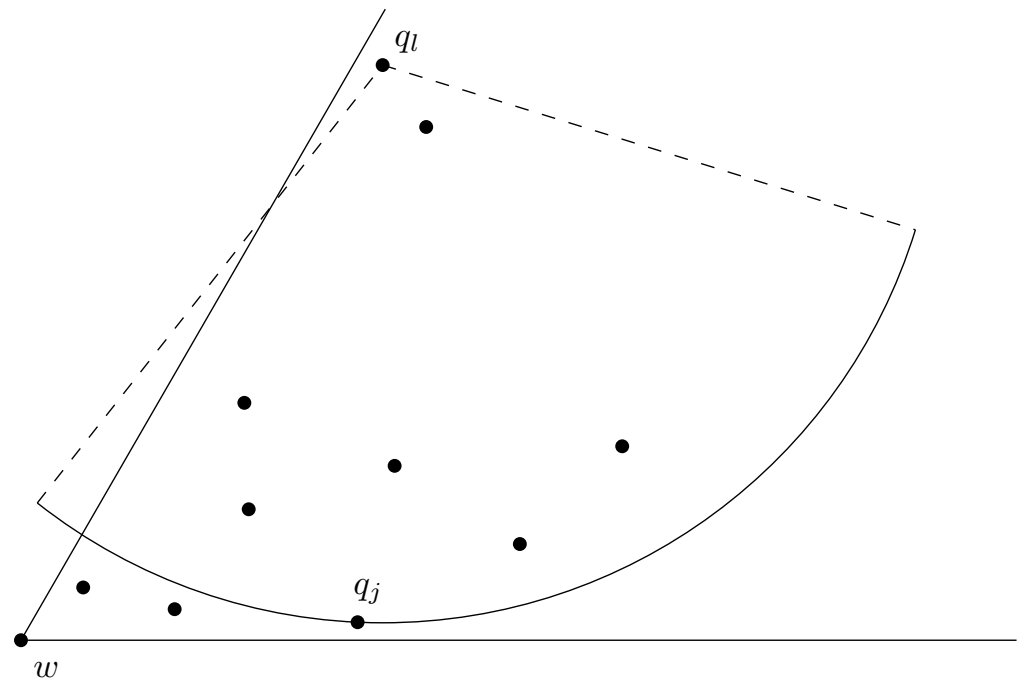

Figure 2: For the proof of Lemma 2

Proof: Let $w \in \mathcal{R}_{i}$ and let $w=q_{0}, q_{1}, q_{2} \ldots q_{k}$ be the points of $\mathcal{R}_{i}$ lying in a wedge of angle $\frac{\pi}{3}$ having apex $w$ sorted by increasing distance to $w$. Clearly, a disk of center $q_{l}$ passing through $q_{j}(j<l)$ cannot contain $w$ (see Figure 2) and thus, if $q=q_{l}$, a necessary condition for $w$ to be in the disk having as diameter the segment defined by $q$ and the nearest neighbor of $q$ in $\mathcal{R}_{i+1}$ is that no point of $\left\{q_{0}, \ldots q_{l-1}\right\}$ is in the sample $\mathcal{R}_{i+1}$. It happens with probability $\left(1-\frac{1}{\alpha}\right)^{l}$.

Using six wedges around $w$ to cover the whole plane, and summing over the choice of $q \in \mathcal{R}_{i}$ we get the claimed result. Notice that the disk of center $q$ and passing through $N N(q)$ contains the disk of diameter $q N N(q)$, thus the expected number of vertices $q$ of $\mathcal{R}_{i}$ belonging to the disk of diameter $q N N(q)$ is less than $6 \alpha$.

Lemma 3 The expected number of edges of $\mathcal{D} \mathcal{T}_{i}$ intersecting segment $q v_{i+1}$ is $O(\alpha)$.

Proof: Let $e$ be an edge of $\mathcal{D} \mathcal{T}_{i}$ intersecting segment $q v_{i+1}$. If $e$ belongs to $\mathcal{D} \mathcal{T}_{\mathcal{R}_{i}}$, it means that $e$ is an internal edge of the region retriangulated when $q$ is inserted in $\mathcal{D} \mathcal{T}_{i}$.

The expected number of such edges is 3 since $q$ is a random point in $\mathcal{R}_{i}$, and that number equals the average degree of $q$ in $\mathcal{R}_{i}$ minus 3 .

If $e$ belongs to $\mathcal{D} \mathcal{T}_{\mathcal{R}_{i}}$, one end-point $w$ of $e$ must belong to the disk of diameter $q v_{i+1}$, denoted $\operatorname{disk}\left[q v_{i+1}\right]$, (otherwise any disk through the end-points of $e$ must contain $q$ or $v_{i+1}$ and $e$ cannot be a Delaunay edge). 
The expected number of edges of $\mathcal{D} \mathcal{T}_{\mathcal{R}_{i}}$ intersecting $\operatorname{disk}\left[q v_{i+1}\right]$ is bounded by the sum of the degrees of the vertices in $\operatorname{disk}\left[q v_{i+1}\right]$

$$
\begin{aligned}
E & \left(\#\left\{e \in \mathcal{D} \mathcal{T}_{\mathcal{R}_{i}} \text { having an end-point } \in \mathcal{R}_{i} \cap \operatorname{disk}\left[q v_{i+1}\right]\right\}\right) \\
& =\frac{1}{\left|\mathcal{R}_{i}\right|} \sum_{q \in \mathcal{R}_{i}} \sum_{w \in \mathcal{R}_{i} \cap \text { disk }\left[q v_{i+1}\right]} d_{\mathcal{D} \mathcal{T}_{\mathcal{R}_{i}}}^{\circ}(w) \\
& =\frac{1}{\left|\mathcal{R}_{i}\right|} \sum_{w \in \mathcal{R}_{i}} d_{\mathcal{D} \mathcal{T}_{\mathcal{R}_{i}}}^{\circ}(w) \cdot \#\left\{q \in \mathcal{R}_{i} \mid w \in \operatorname{disk}\left[q v_{i+1}\right]\right\} \\
& \leq \frac{1}{\left|\mathcal{R}_{i}\right|} \sum_{w \in \mathcal{R}_{i}} d_{\mathcal{D}_{\mathcal{R}_{i}}}^{\circ}(w) 6 \alpha \quad \text { using Lemma } 2 \\
& \leq 36 \alpha \text { using the bound of } 6 \text { on the average degree of } w
\end{aligned}
$$

Notice that Lemma 2 was established for a fixed $w$ and a random $q$ which allows it to be used inside the sum over $w$. Thus we get a total expected cost for the walk bounded by $36 \alpha+3$.

Lemma 4 The expected number of triangles of $\mathcal{D} \mathcal{T}_{i}$ visited during the search for $v_{i}$ from $t_{i}$ is $O(\alpha)$.

Proof: The triangles examined in Phase 3, either are triangles of $\mathcal{D} \mathcal{T}_{i} \backslash \mathcal{D} \mathcal{T}_{\mathcal{R}_{i}}$ and the expected number of such triangles is 4 , or are triangles of $\mathcal{D} \mathcal{T}_{i} \cap \mathcal{D} \mathcal{T}_{\mathcal{R}_{i}}$ and have a vertex in the disk of center $q$ passing through $v_{i+1}$. Thus we can argue as in Lemma 3, denoting disk $\left.\left.\right|_{c} q v_{i+1}\right]$ the disk of center $q$ through $v_{i+1}$ :

$$
\begin{aligned}
E & \left.\left(\#\left\{t \in \mathcal{D} \mathcal{T}_{\mathcal{R}_{i}} \text { having a vertex }\left.\in \operatorname{disk}\right|_{c} q v_{i+1}\right]\right\}\right) \\
& \leq \frac{1}{\left|\mathcal{R}_{i}\right|} \sum_{q \in \mathcal{R}_{i}} \sum_{w \in \mathcal{R}_{i} \cap \text { disk }} d_{\left.{ }_{c} q v_{i+1}\right]}^{\circ} d_{\mathcal{D} \mathcal{T}_{\mathcal{R}_{i}}}(w) \\
& \left.\leq \frac{1}{\left|\mathcal{R}_{i}\right|} \sum_{w \in \mathcal{R}_{i}} d_{\mathcal{D T}_{\mathcal{R}_{i}}^{\circ}}^{\circ}(w) \mid\left\{q \in \mathcal{R}_{i}\left|w \in \mathcal{R}_{i} \cap \operatorname{disk}\right|{ }_{c} q v_{i+1}\right]\right\} \mid \\
& \leq \frac{1}{\left|\mathcal{R}_{i}\right|} \sum_{w \in \mathcal{R}_{i}} d_{\mathcal{D T}_{\mathcal{R}_{i}}}^{\circ}(w) 6 \alpha \quad \text { using Lemma } 2 \\
& \leq 36 \alpha \quad \text { using the bound on the average degree of } w
\end{aligned}
$$

The expected number of visited triangles is less than $4+36 \alpha$. $\diamond$ An alternative solution for Phase 3 consists of simulating the insertion of $q$ in $\mathcal{D} \mathcal{T}_{i}$ in order to select all the points which are neighbors of $q$ in $\mathcal{D} \mathcal{T}_{\mathcal{R}_{i}}$. Since we know that $q$ and $v_{i}$ are neighbors in $\mathcal{D} \mathcal{T}_{\mathcal{R}_{i}}, v_{i}$ can be selected from these vertices. In that case the visited triangles are those of $\mathcal{D} \mathcal{T}_{i} \backslash \mathcal{D} \mathcal{T}_{\mathcal{R}_{i}}$ plus their neighbors, that is, an expected number of less than 10. This approach involves more complicated tests than the approach analyzed above. 
Theorem 5 The expected cost of inserting the $n^{\text {th }}$ point in the Delaunay hierarchy is $O\left(\alpha \log _{\alpha} n\right)$

Proof: By linearity of expectation, Lemmas 1, 3 and 4 prove that the expected cost at one level is $O(\alpha)$. Since the expected height of the structure is $\log _{\alpha} n$, we get the claimed result. (The analysis is similar to the analysis for skip lists [23].)

Theorem 6 The construction of the Delaunay hierarchy of a set of $n$ points is done in expected time $O\left(\alpha n \log _{\alpha} n\right)$ and $O\left(\frac{\alpha}{\alpha-1} n\right)$ space. The expectation is on the randomized sampling and the order of insertion, with no assumption on the point distribution.

Proof: Easy corollary of Theorem 5.

\section{Higher dimensional case}

The previous algorithm and its analysis generalize easily to higher dimensions. The algorithm uses several levels of samples. At level $i$ it uses a walking strategy to locate the simplex containing a query, then its nearest neighbor $v_{i}$ can be found by simulating the insertion of $q$ in $\mathcal{D} \mathcal{T}_{i}$.

The analysis of the previous section uses essentially the fact that the nearest neighbor graph is of bounded degree 6 and the fact that the expected degree of a point in a triangulation is also 6 . The cost of walking at level $i$ is the product of these two numbers. In higher dimensions, the degree of the nearest neighbor graph remains bounded by some constant depending on the dimension. Unfortunately the expected degree of a random point is not bounded in higher dimensions. As usual in analysis of incremental randomized constructions [4], we can write aexpress the complexity as sensitive to the expected size of the Delaunay triangulation of a sample. If $f(r)$ is the expected size of the Delaunay triangulation of a random sample of size $r$ of $\mathcal{S}$, then the expected degree of a random point in the triangulation of that sample is $\frac{f(r)}{r}$.

Lemmas 1, 2, 3 and 4 generalize easily: Lemma 1 gives that the expected degree of $v_{i}$ is $O\left(\frac{f(r)}{r}\right)$. Lemma 2 remains unchanged (up to a constant) and Lemmas 3 and 4 give bounds of $O\left(\alpha \frac{f(r)}{r}\right)$ for the Phases 2 and 3 of the walk. The expected complexity of walking at level $i$ is $O\left(\alpha \frac{f\left(\alpha^{i} n\right)}{\alpha^{i} n}\right)$. Summing on the different levels yields an expected complexity of $\sum_{i=0}^{\infty} \alpha \frac{f\left(\alpha^{i} n\right)}{\alpha^{i} n}$ to locate a point in the Delaunay hierarchy.

There exist several bounds for $f(r)$; in the worst case $f(r)=r^{\left\lceil\frac{d}{2}\right\rceil}$ and we get Theorems 7 and 8 below. If the points are evenly distributed, $f(r)=O(r)$ [18] and the complexity of inserting a point is logarithmic. Other results on $f(r)$ exist, for example if there is a minimal distance between points [19] or if the points belong to a surface [2]. 
Theorem 7 The expected cost of inserting the $n^{\text {th }}$ point in a d-dimensional Delaunay hierarchy for any constant ratio $\alpha$ is $O\left(n^{\left\lceil\frac{d-2}{2}\right\rceil}\right)$.

Proof: The expected cost at level $i$ is bounded by the maximum degree of the nearest neighbor graph multiplied by $\alpha\left(\alpha^{i} n\right)^{\left\lceil\frac{d-2}{2}\right\rceil}$, summing for $i$ going from 0 to $\infty$ gives $O\left(\frac{\alpha}{\left.1-\alpha\rceil \frac{d-2}{2}\right\rceil} n^{\left\lceil\frac{d-2}{2}\right\rceil}\right)$.

Theorem 8 The construction of the d-dimensional Delaunay hierarchy of a set of $n$ points can be done in expected time and space $O\left(n^{\left\lceil\frac{d}{2}\right\rceil}\right)$. The expectation is on the randomized sampling and the order of insertion, with no assumption on the point distribution.

Proof: Easy corollary of Theorem 7.

\section{Tuning parameters}

We have proved that our structure has an asymptotically worst case optimal $\operatorname{cost} O(n \log n)$ for any set of points in the plane. The constant hidden in the $O$ actually varies with $\alpha$, the ratio between two levels of the hierarchy. In this section, we propose a more precise analysis, not in the worst case, but for points evenly distributed in a square ${ }^{1}$ to tune the parameter $\alpha$ in that case.

This section needs a careful analysis of the constant involved in the different phases of the algorithm which implies a precise counting of the arithmetic operations involved.

\subsection{Phase 1}

We can assume that $d_{\mathcal{D} \mathcal{T}_{i}}^{\circ}\left(v_{i+1}\right)=6$ (and not only $\leq 36$ as proved in Lemma 1$)$. And thus if the turn around $v_{i+1}$ is clockwise or counterclockwise depending on the position of segment $v_{i+1} q$ with respect to the starting triangle, and assuming that this position is random around $v_{i+1}$, the expected number of orientation tests $^{2}$ is 3 . Figure 3 shows the different cases to average; the edges $v_{i+1} w$ such that an orientation test $v_{i+1} w q$ is performed are indicated for a typical degree 6 vertex in the triangulation.

\footnotetext{
${ }^{1}$ We denote by $\gamma$ the point density, that is, the expected number of points in any unit square.

${ }^{2}$ An orientation test takes three points $p, q$ and $r$ and reports if the triangle is clockwise, counterclockwise or degenerate.
} 


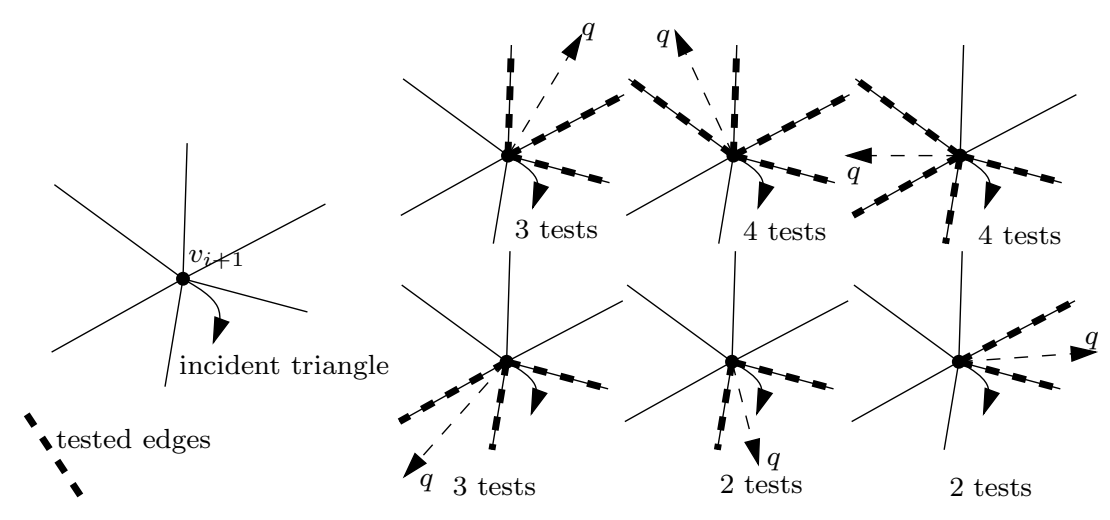

Figure 3: Number of orientation tests in Phase 1

\subsection{Phase 2}

Devroye et al. $[17,7]$ proved that the expected number of edges of a Delaunay triangulation of random points crossed by a line segment of length $l$ is $O(l \sqrt{\gamma})$ where $\gamma$ is the point density. Our experiments show that the constant is 2 .

The expected number of points in the disk of center $q$ passing through $v_{i+1}$ is $\alpha-1$. Indeed, if the points of $\mathcal{R}_{i}$ are sorted by increasing distance from $q$, $v_{i+1}$ is the first point in $\mathcal{R}_{i+1}$, thus the number of points in the disk is $k$ with probability $\left(1-\frac{1}{\alpha}\right)^{k} \frac{1}{\alpha}$ (probability that the $k$ first points are not in the sample and the $k+1^{\text {st }}$ point is), and the expected number is $\frac{1}{\alpha} \sum k\left(1-\frac{1}{\alpha}\right)^{k}=\alpha-1$. Thus if $l$ is the length of $q v_{i+1}$ the density of points in $\mathcal{D} \mathcal{T}_{i}$ is $\gamma=\frac{\alpha}{\pi l^{2}}$.

Thus we conclude that the expected number of edges of $\mathcal{D} \mathcal{T}_{i}$ intersecting segment $q v_{i+1}$ is $i 2 l \sqrt{\gamma}=2 l \sqrt{\frac{\alpha}{\pi l^{2}}}=\frac{2 \sqrt{\alpha}}{\sqrt{\pi}}$.

For each edge $w w^{\prime}$ crossed, two orientation tests are performed [14]: if $w$ is the newly examined vertex, orientations of triangles $w q v_{i+1}$ and $q w w^{\prime}$ are computed.

\subsection{Phase 3}

Phase 3 is more difficult to analyze precisely, but a rough bound is that the number of candidate vertices examined (with shortest distance) is less than two and that we examine less than 8 triangles in total.

In fact, we modified Phase 3 so that instead of really searching for $v_{i}$, the nearest neighbor of $q$ in $\mathcal{S}_{i}$, we just define $v_{i}$ as the nearest among the three vertices of $t_{i}$. This modified Phase 3 is reduced to three distance computations and two comparisons. 


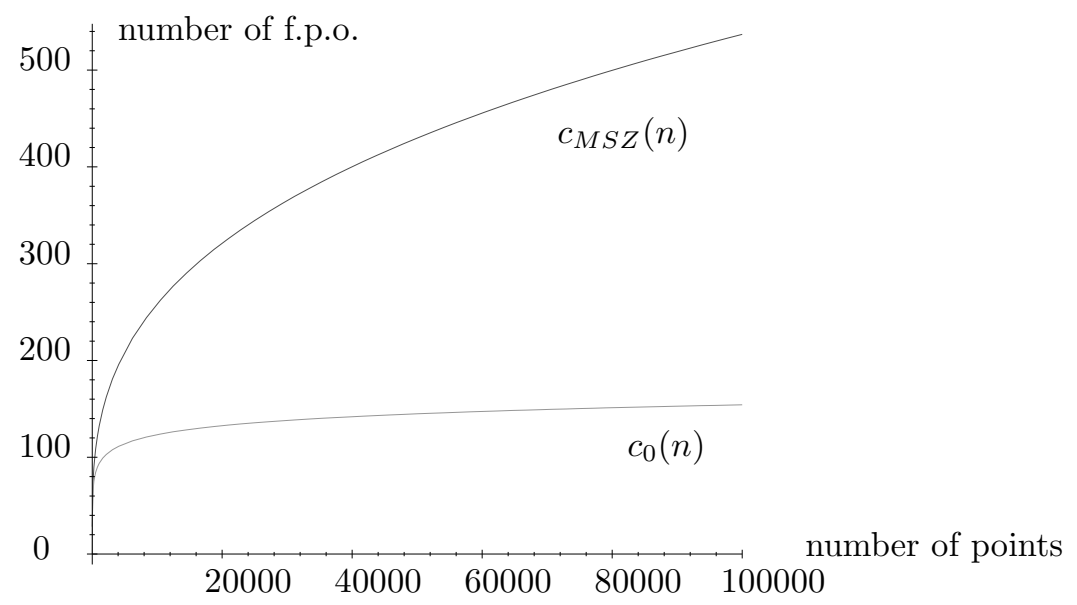

Figure 4: Comparison of the number of floating point operations between $c_{0}(n)$ and $c_{M S Z}(n)$ for $\alpha=40$ and $\beta=1$.

\subsection{Tuning $\alpha$}

A precise counting [10] of floating point operations (f.p.o.) yields a complexity of $36+6.2 \sqrt{\alpha}$ at each level. Since the number of levels is $\log _{\alpha} n=\frac{\log _{2} n}{\log _{2} \alpha}$ we get a cost of $c_{0}(n)=(32+6.2 \sqrt{\alpha})\left[\frac{\log _{2} n}{\log _{2} \alpha}\right]$ which is close to its minimum ( $\left.c_{0}(n) \in\left[13.3 \log _{2} n, 14 \log _{2} n\right]\right)$ for $\alpha \in[18,90]$, with the minimum occuring when $\alpha \simeq 40$.

\subsection{Comparison with [24]}

A similar counting [10] of f.p.o. in the jump and walk algorithm, using a random sample of $\beta \sqrt[3]{n}$ points, produces a cost of $c_{M S Z}(n)=17+\sqrt[3]{n}\left(\frac{6.2}{\sqrt{\beta}}+5 \beta\right)$ which is close to its minimal value for $0.5<\beta<1$.

As shown by the comparison of the two curves in Figure 4, our method is potentially much better than jump and walk [24], even for a small number of points. However, this method of analyzing our approach hides the discontinuity of the cost, since the effective number of levels is necessarily an integer. To have a better understanding of what happens for a small number of points, we plot the cost of inserting a point in a structure having a fixed number of levels.

The classical walk from a random point in the structure costs $c_{\text {walk }}(n)=$ $17+6.2 \sqrt{n}$ which is also the cost of inserting in our structure up to the time a second level is created. 
When $k$ levels have been created, the cost is

$$
c_{k}(n)=c_{w a l k}\left(\frac{n}{\alpha^{k}}\right)+15 k+k \cdot c_{w a l k}(\alpha) .
$$

We can alternatively mix this multilevel approach with Mücke et al's. sampling at the first level of the structure. In that case, the cost is

$$
c_{k}^{\star}(n)=c_{M S Z}\left(\frac{n}{\alpha^{k}}\right)+15 k+k \cdot c_{w a l k}(\alpha) .
$$

This comparison (see Figure 5) shows that jump and walk [24] $\left(c_{1}^{\star}(n)\right)$ becomes better than the simple walk $\left(c_{1}(n)\right)$ for $n>40$. The two level delaunay hierarchy $\left(c_{2}(n)\right)$ becomes better than the simple walk $\left(c_{1}(n)\right)$ for $n>180$ and better than jump and walk [24] $\left(c_{1}^{\star}(n)\right)$ for $n>600$. The main information is that our structure should be significantly better than [24] for $10000<n$.

\section{Implementation}

\subsection{Deletion}

The above structure supports insertions and queries as explained above, but also deletions. Since there is no complicated data structure to maintain, deletions can be handled by just deleting the removed point at each level where it appears.

This can be done in output-sensitive time $[9,1]$, and thus the deletion of a random point is done in expected constant time since a point appears at an expected constant number of levels and its expected degree $k$ is also constant.

From a practical point of view, and to keep the simplicity of the algorithm, a simpler suboptimal algorithm is better. Point deletion can be done in $O(k \log k)$ time in any dimension [12]. The hole created by the removal of all faces incident to the removed points is filled by adding the new simplices in some good order.

\subsection{Arithmetic degree}

A basic geometric test (also called predicate) such as the orientation test usually reduces to the evaluation of the sign of some polynomial; the degree of such a polynomial is a useful measure of the complexity of the predicates used by the algorithm [22]. The algorithm above is designed to make a parsimonious use of high degree tests. More precisely, the location phase uses only orientation tests on three points in Phases 1 and 2, and distance computations and angle comparisons with $\frac{\pi}{2}$ in Phase 3. All these tests have degree 2. Updating the Delaunay triangulation clearly needs to use in-circle tests which are of degree 4 .

In higher dimensions, Phases 1 and 2 use degree $d$ tests; Phase 3 and point insertion use degree $d+2$ tests. 


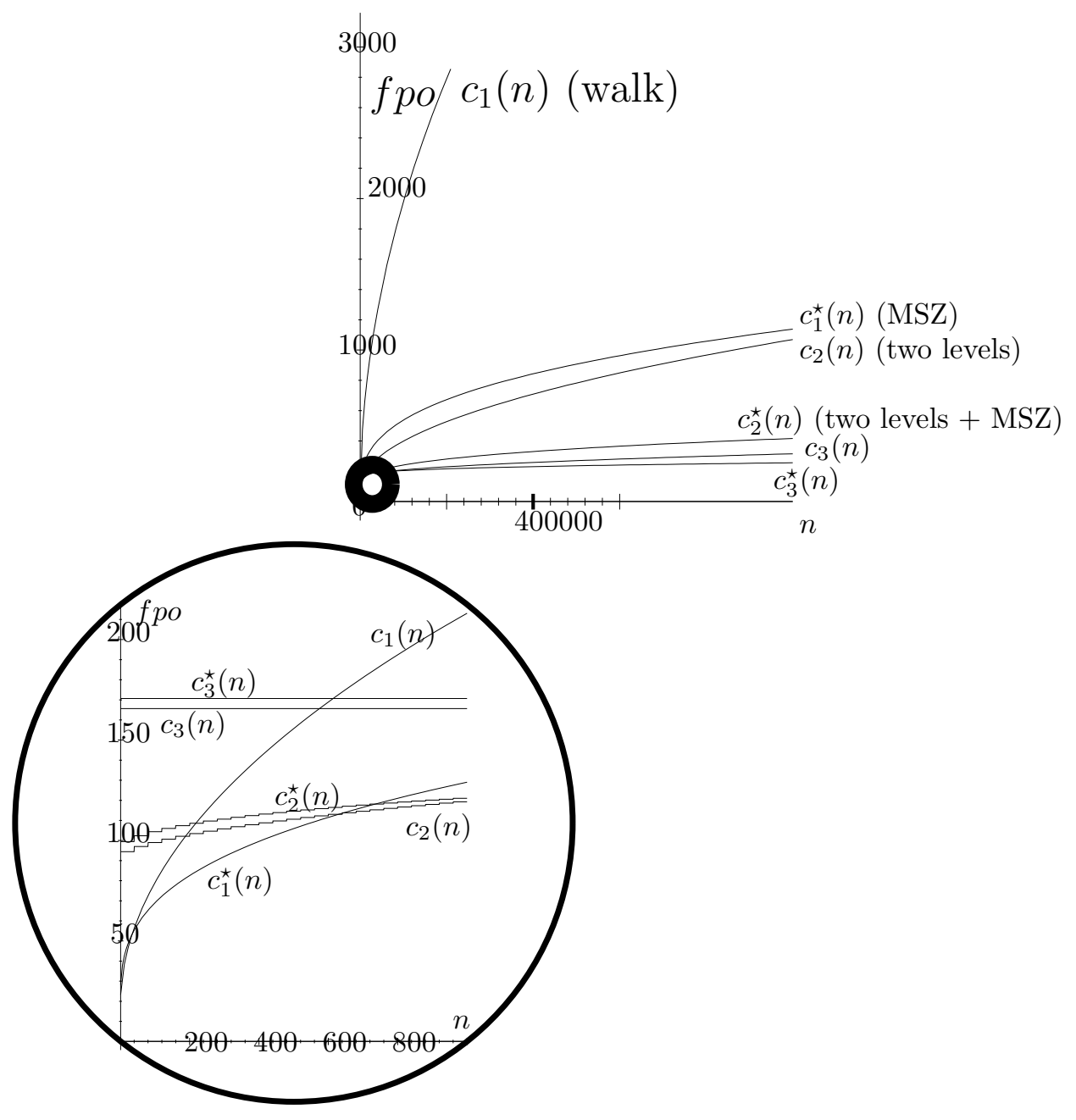

Figure 5: Comparison of the number of floating point operations between $c_{k}(n)$ and $c_{k}^{\star}(n)$ for $\alpha=40$. 


\subsection{Robustness issues and degeneracies}

Degeneracies are solved by handling special cases: if two points have the same coordinates, then the insertion is not done, if four points are cocircular, then the last point inserted is considered as outside the disk defined by the others. This corresponds to the standard perturbation scheme [28] on the convex hull of the points projected on the paraboloid in 3 dimensions. This generalizes in any dimension.

We use exact arithmetic for 24 bits integers, and thus the coordinates of our points are integers in range $[-16.777 .216,16.777 .216]$ (up to a multiplication by a power of 2). Using this restricted kind of number, double precision computation is exact on degree 2 tests and almost never leads to precision problems on degree 4 predicates. Nevertheless, the exactness of all computations are verified by an arithmetic filter and exact computation is performed if needed.

In dimension three, we use the same kind of numbers. The orientation test has degree 3 and the in sphere test have degree 5. All computations are done using filtered floating point computations and exact computation if needed $[15,16]$.

\subsection{Experimental results}

We have investigated the performance of our algorithm in different situations.

All point sets have 1.000 .000 points (except moment), times are the time ${ }^{3}$ for reading the points and computing the Delaunay triangulation in seconds.

\subsubsection{Sensibility to the input distribution}

We have used the different kinds of input distributions described below:

2D

$$
\text { square }
$$

ellipse

mixed

parabola

circle

$3 \mathrm{D}$ points evenly distributed in a square points evenly distributed on an ellipse 95\% points evenly distributed on an ellipse plus $5 \%$ points evenly distributed in a square points evenly distributed on the parabola $y=x^{2}$ and rounded to 24 bits

cube points evenly distributed in a cube

ellipsoid

object points evenly distributed on an ellipsoid

sphere points measured on an object with a $3 \mathrm{D}$ sensor

moment points evenly distributed on a sphere (rounded) points evenly distributed on the moment curve $y=x^{2}, z=x^{3}$ (rounded), 5.000 points only

\footnotetext{
${ }^{3}$ Running times have been obtained on a PentiumIII $730 \mathrm{MHz} 640 \mathrm{Mo}$. Code is in $\mathrm{C}++$ compiled with g++ under Linux.
} 
The following table gives the execution times. We can observe that the running times are of the same order for the different kinds of distribution (except for the quadratic example in 3D). By playing with the parameters, we can use the same software to use the Hierarchy with or without the cubic root sampling of Mcke, Saias and Zhu, the cubic root sampling without the hierarchy, or a simple walk in the triangulation. The Delaunay hierarchy runs much faster than the cubic root sampling in two dimensions. In three dimensions, the advantage is less important but still significant, especially on some non uniform distributions.

\begin{tabular}{|l|r|r|r|r|}
\hline data & $\begin{array}{r}\text { Hierarchy } \\
+ \text { sampling }\end{array}$ & $\begin{array}{r}\text { Hierarchy } \\
\text { only }\end{array}$ & $\begin{array}{r}\text { cubic root } \\
\text { sampling }\end{array}$ & walk \\
\hline square & 19 & 20 & 56 & 488 \\
ellipse & 28 & 35 & 106 & 3380 \\
mixed & 31 & 31 & 708 & 392 \\
parabola & 33 & 35 & 170 & \\
circle & 22 & 22 & 23 & 22 \\
\hline cube & 90 & 94 & 102 & 245 \\
ellipsoid & 95 & 98 & 197 & 774 \\
object & 88 & 88 & 108 & 174 \\
sphere & 71 & 72 & 72 & 85 \\
moment, 5000 & 62 & 62 & 63 & 71 \\
\hline
\end{tabular}

\subsubsection{Sensibility to the ratio between levels}

The table below shows the running time for different point distributions and depending of the ratio used to sample the levels. The line square in this table agrees with the theoretical results of Section 5.4 that the behavior of the algorithm should be good for a ratio between levels between 18 and 90; this validates our counting of floating point operations as a relevant measure to establish this optimal value of the ration $\alpha$. . For non uniform distributions, the table shows that a small ratio in the interval $[18,90]$ should be prefered. In all other tables, we choose a ratio of 30 . Using this ratio of 30 and for one million points, the hierarchy has usually 3 levels of samples.

\begin{tabular}{|l|r|r|r|r|r|r|r|r|}
\hline ratio between levels & 3 & 5 & 10 & 20 & 30 & 80 & 200 & 500 \\
\hline square & 27 & 23 & 20 & 19 & 19 & 21 & 24 & 27 \\
mixed & 29 & 25 & 24 & 28 & 31 & 47 & 75 & 113 \\
ellipse & 27 & 25 & 24 & 26 & 27 & 37 & 53 & 46 \\
\hline cube & 147 & 123 & 106 & 100 & 97 & 96 & 97 & 98 \\
object & 130 & 110 & 95 & 89 & 89 & 87 & 89 & 91 \\
\hline
\end{tabular}

\subsubsection{Sensibility to randomness}

The complexity is randomized, that is if you are unlucky with the random sample or the random order of insertion the running time may increase. As shown by the following table which gives the running time of different execution 
with different choices for the random sampling, the variance of the expected running time of the algorithm is in fact very low and running times have a variation of less than $1 \%$. Since the times vary within this interval of $1 \%$ for different runs of the algorithm, times in other tables have been rounded to the precision of the second.

\begin{tabular}{|c|c|c|c|c|c|}
\hline mixed & 30.8 & 30.8 & 31.4 & 31.3 & 31.0 \\
\hline object & 88.3 & 87.2 & 87.9 & 88.6 & 86.9 \\
\hline
\end{tabular}

\subsubsection{Comparison with other software}

We have compared the two dimensional version with some Delaunay software available on the WWW:

- qhull by Bradford Barber and Hannu Huhdanpaa, duality with 3D convex hull [3] (available at http://www.geom.umn.edu/locate/qhull). This algorithm is static.

- div-conquer by Jonathan Shewchuk, divide and conquer [29] algorithm using a quadtree scheme. This algorithm is static.

- sweep by Jonathan Shewchuk, implements Fortune's plane sweep algorithm, which is static.

- incremental by Jonathan Shewchuk, incremental with Mücke et al. localization, this algorithm is semi-dynamic and can be easilly modified to be fully dynamic.

These three codes support exact arithmetic on double (available at http://www.cs.cmu.edu/ quake/triangle.research.html).

- Dtree Delaunay tree structure[6] provides a semi-deynamic algorithm (available at http://www.inria.fr/prisme/logiciel/del-tree.html).

- hierarchy this paper.

The execution times ${ }^{4}$ in seconds are in the table below. Our method is significantly faster than the other incremental method, specially in the ellipse cases. Our method is about $50 \%$ slower than the divide and conquer algorithm and is clearly the best fully dynamic algorithm.

\footnotetext{
${ }^{4}$ This set of runs have been done on a Sun-Ultra1 $200 \mathrm{MHz} 128 \mathrm{Mo}$.
} 


\begin{tabular}{|l|r||r|r|r|r|r|r|}
\hline distribution & size & qhull & sweep & div-conq & incr & Dtree & hierarchy \\
\hline \hline random & 5000 & 0.65 & 0.21 & 0.11 & 0.29 & 1.4 & 0.14 \\
\hline random & 50000 & 8.0 & 3.6 & 1.6 & 6.6 & 17 & 2.3 \\
\hline random & 500000 & 101 & 53 & 22 & 150 & swap & 31 \\
\hline \hline mixed & 5000 & 0.54 & 0.21 & 0.13 & 0.75 & 1.3 & 0.20 \\
\hline mixed & 50000 & 7.8 & 3.2 & 2.16 & 42 & 16 & 3.5 \\
\hline mixed & 500000 & 420 & 46 & 29 & 2100 & swap & 49 \\
\hline \hline ellipse & 5000 & 0.83 & 0.18 & 0.14 & 2.1 & 1.3 & 0.21 \\
\hline ellipse & 50000 & 57 & 2.8 & 2.4 & 110 & 14 & 3.7 \\
\hline ellipse & 500000 & swap & 39 & 33 & 1400 & swap & 55 \\
\hline \hline parabola & 5000 & 3.9 & 0.16 & 0.11 & 2.0 & 1.2 & 0.16 \\
\hline parabola & 50000 & 790 & 2.7 & 2.0 & 110 & 14 & 3.0 \\
\hline parabola & 500000 & swap & 39 & 28 & 1800 & swap & 45 \\
\hline \hline circle & 5000 & 93 & 0.17 & 0.17 & 0.52 & 1.4 & 0.14 \\
\hline circle & 50000 & 220 & 3.1 & 1.8 & 11 & 15 & 2.4 \\
\hline circle & 500000 & swap & 22 & 43 & 240 & swap & 36 \\
\hline \hline
\end{tabular}

\section{Conclusion}

We have proposed a new hierarchical data structure to compute the Delaunay triangulation of a set of points in the plane. It combines good worst case randomized complexity, fast behavior on real data, small memory occupation and dynamic updates (insertion and deletion of points).

Referring to Su and Drysdale's study of several techniques [30] and our comparisons with Shewchuk implementations [29] of some of these techniques, we have shown that our implementation is competitive with other approaches on random data; the only faster approach for large inputs is the divide and conquer algorithm which is not dynamic. Furthermore, we can prove that the performance remains good on pathological inputs.

The main idea of our structure is to perform point location using several levels. The lowest level just consists of the triangulation, then each level contains the triangulation of a small sample of the levels below. Point location is done by walking along a line segment in a triangulation to determine the nearest neighbor of the query at that level, then the walk restarts from that neighbor at the level below. Other walking strategies have been proposed [14] and may be more efficient in practice but there is no theoretical result on the complexity of these alternative walks. Location at the highest level is done using jump and walk [24] which is efficient for small sets of points.

One characteristic of the Delaunay hierarchy is that the best time performance is obtained with a ratio of about three per cent between two levels and thus a very small number of levels (three or four typically) and thus good performances in terms of memory occupation. The structure is simple and does 
not need additional features such as buckets.

Such structures can be generalized to other problems. The two main ingredients of the proofs are bounds on the maximal degree of the nearest neighbor graph and the expected degree of a random vertex in the Delaunay triangulation. The first generalizes well in higher dimensions, while the second becomes a data sensitive parameter (constant for random points, $n^{\lceil(d-1) / 2\rceil}$ in the worst case). A generalization for computing the trapezoidal map can also be done.

\section{Code}

A demo version compiled for Sun Solaris is available at http://www.inria.fr/prisme/logiciels/del-hierarchy/.

\section{Acknowledgements}

The author would like to thank Herv Brnnimann, Hazel Everett, Jean-Michel Moreau, Jonathan Shewchuk, Jack Snoeyink and Mariette Yvinec for helpful discussions.

\section{References}

[1] A. Aggarwal, L. J. Guibas, J. Saxe, and P. W. Shor. A linear-time algorithm for computing the Voronoi diagram of a convex polygon. Discrete Comput. Geom., 4(6):591-604, 1989.

[2] Dominique Attali and Jean-Danial Boissonnat. Complexity of the delaunay triangulation of points on a smooth surface. Research Report, to appear, INRIA, 2001.

[3] C. B. Barber, D. P. Dobkin, and H. Huhdanpaa. The Quickhull algorithm for convex hull. Technical Report GCG53, Geometry Center, Univ. of Minnesota, July 1993.

[4] Jean-Daniel Boissonnat, Olivier Devillers, René Schott, Monique Teillaud, and Mariette Yvinec. Applications of random sampling to on-line algorithms in computational geometry. Discrete Comput. Geom., 8:51-71, 1992.

[5] Jean-Daniel Boissonnat and Monique Teillaud. A hierarchical representation of objects: The Delaunay tree. In Proc. 2nd Annu. ACM Sympos. Comput. Geom., pages 260-268, 1986.

[6] Jean-Daniel Boissonnat and Monique Teillaud. On the randomized construction of the Delaunay tree. Theoret. Comput. Sci., 112:339-354, 1993. 
[7] P. Bose and L. Devroye. Intersections with random geometric objects. Technical report, School of Computer Science, McGill University, 1995. Manuscript.

[8] L. P. Chew. Building Voronoi diagrams for convex polygons in linear expected time. Technical Report PCS-TR90-147, Dept. Math. Comput. Sci., Dartmouth College, Hanover, NH, 1986.

[9] L. P. Chew. Constrained Delaunay triangulations. In Proc. 3rd Annu. ACM Sympos. Comput. Geom., pages 215-222, 1987.

[10] O. Devillers. Improved incremental randomized Delaunay triangulation. Research Report 3298, INRIA, 1997.

[11] Olivier Devillers. Improved incremental randomized Delaunay triangulation. In Proc. 14th Annu. ACM Sympos. Comput. Geom., pages 106-115, 1998.

[12] Olivier Devillers. On deletion in Delaunay triangulation. In Proc. 15th Annu. ACM Sympos. Comput. Geom., pages 181-188, 1999.

[13] Olivier Devillers, Stefan Meiser, and Monique Teillaud. Fully dynamic Delaunay triangulation in logarithmic expectedtime per operation. Comput. Geom. Theory Appl., 2(2):55-80, 1992.

[14] Olivier Devillers, Sylvain Pion, and Monique Teillaud. Walking in a triangulation. This volume.

[15] Olivier Devillers and Franco P. Preparata. A probabilistic analysis of the power of arithmetic filters. Discrete Comput. Geom., 20:523-547, 1998.

[16] Olivier Devillers and Franco P. Preparata. Further results on arithmetic filters for geometric predicates. Comput. Geom. Theory Appl., 13:141-148, 1999 .

[17] Luc Devroye, Ernst Peter Mücke, and Binhai Zhu. A note on point location in Delaunay triangulations of random points. Algorithmica, 22:477-482, 1998.

[18] R. A. Dwyer. Higher-dimensional Voronoi diagrams in linear expected time. Discrete Comput. Geom., 6:343-367, 1991.

[19] Jeff Erickson. Nice point sets can have nasty Delaunay triangulations. In Proc. 17th Annu. ACM Sympos. Comput. Geom., pages 96-105, 2001.

[20] Leonidas J. Guibas, D. E. Knuth, and Micha Sharir. Randomized incremental construction of Delaunay and Voronoi diagrams. Algorithmica, 7:381413,1992 . 
[21] C. L. Lawson. Software for $C^{1}$ surface interpolation. In J. R. Rice, editor, Math. Software III, pages 161-194. Academic Press, New York, NY, 1977.

[22] Giuseppe Liotta, Franco P. Preparata, and Roberto Tamassia. Robust proximity queries: An illustration of degree-driven algorithm design. SIAM J. Comput., 28(3):864-889, 1998.

[23] R. Motwani and P. Raghavan. Randomized Algorithms. Cambridge University Press, New York, NY, 1995.

[24] Ernst P. Mücke, Isaac Saias, and Binhai Zhu. Fast randomized point location without preprocessing in two- and three-dimensional Delaunay triangulations. In Proc. 12th Annu. ACM Sympos. Comput. Geom., pages 274-283, 1996.

[25] K. Mulmuley. Randomized multidimensional search trees: Dynamic sampling. In Proc. 7th Annu. ACM Sympos. Comput. Geom., pages 121-131, 1991.

[26] K. Mulmuley. Computational Geometry: An Introduction Through Randomized Algorithms. Prentice Hall, Englewood Cliffs, NJ, 1993.

[27] M. S. Paterson and F. F. Yao. On nearest-neighbor graphs. In Proc. 19th Internat. Colloq. Automata Lang. Program., volume 623 of Lecture Notes Comput. Sci., pages 416-426. Springer-Verlag, 1992.

[28] R. Seidel. The nature and meaning of perturbations in geometric computing. In Proc. 11th Sympos. Theoret. Aspects Comput. Sci., volume 775 of Lecture Notes Comput. Sci., pages 3-17. Springer-Verlag, 1994.

[29] J. R. Shewchuk. Triangle: Engineering a 2d quality mesh generator and Delaunay triangulator. In First Workshop on Applied Computational Geometry. Association for Computing Machinery, May 1996.

[30] P. Su and R. Drysdale. A comparison of sequential Delaunay triangulation algorithms. Comput. Geom. Theory Appl., 7:361-386, 1997. 